\title{
Comparison of Iron Oxide-Related MRI Artifacts in Healthy and Neuropathological Human Brain Tissue
}

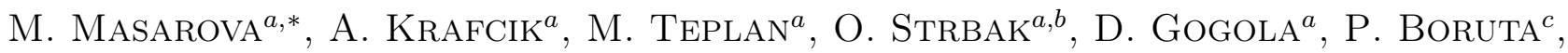 \\ D. DOBROTA ${ }^{b}$ AND I. FROLlO ${ }^{a}$ \\ ${ }^{a}$ Institute of Measurement Science, SAS, Dubravska cesta 9, 84105 Bratislava, Slovakia \\ ${ }^{b}$ Biomedical Center Martin, Jessenius Faculty of Medicine in Martin, Comenius University in Bratislava, \\ Mala Hora 4, 03601 Martin, Slovakia \\ ${ }^{c}$ Slovak Medical University, Limbova 12, 83303 Bratislava, Slovakia
}

\begin{abstract}
The aim of this study is to clarify whether clinical magnetic resonance imaging data can be utilised to evaluate the pathological processes associated with disrupted iron homeostasis, such as neurodegenerative processes or cirrhosis. Although MRI has the potential to become a non-invasive biomarker of such pathology, new quantification methods must be introduced. Our findings confirmed that it is possible to detect significant difference between healthy and pathological tissue from standard $T 2$ weighted MRI protocols.
\end{abstract}

DOI: $10.12693 /$ APhysPolA.131.1108

PACS/topics: 87.61.Ff, 75.50.Cc, 87.19.xr, 87.19.xe

\section{Introduction}

Iron is an essential nutrient required by every human cell, with the iron in the human body having two major forms: heme and non-heme iron. Approximately $80 \%$ of body iron is functional, located in red blood cells as haemoglobin, in muscles as myoglobin; it also forms part of iron-containing enzymes. Non-heme iron can be found in transporter molecules such as transferrin, as well as in storage molecules such as ferritin and hemosiderin. Transferrin is an intravascular transport protein that delivers iron to the liver, bone marrow, and other tissues. Ferritin is a spherical protein shell which is able to accumulate and store up to 4500 iron atoms [1, 2].

Iron plays an important role in the central nervous system, where it is involved in the transportation of oxygen, neurotransmission, and myelin production and maintenance [1]. The diameters of the ferritin iron cores in the brain are smaller than those in the liver $(3.5 \pm 0.5 \mathrm{~nm}$ vs. $6.0 \pm 0.5 \mathrm{~nm}$ ) [2]. The abnormal accumulation of iron or disruption of the iron metabolism has been detected in various liver and neurodegenerative illnesses such as the Parkinson disease, multiple sclerosis, and the Alzheimer disease [1]; however, iron levels in the brain also increase with age [3]. Tissue iron can promote oxidative damage by catalysing free-radical reactions, resulting in the formation of hydroxyl radicals which denature protein and DNA and initiate lipid peroxidation.

Studies of bulk brain iron have reported increased iron levels in the brains of those with the Alzheimer disease [3]. The distribution of non-heme iron is not uniform in all areas of the brain, but varies depending on the

*corresponding author; e-mail: marta.masarova@savba.sk brain structure. In healthy subjects, the largest concentrations estimated from postmortem staining are located in the basal ganglia, with the globus pallidus having the highest concentrations at any age. The red nucleus and substantia nigra also contain relatively large amounts of iron, although not to the extent of the basal ganglia, with even smaller iron content observed in other subcortical nuclei such as the thalamus [1, 3-5].

Histological study suggests that ferritin and hemosiderin are the only paramagnetic materials of sufficient concentration to affect the magnetic resonance (MR) signal from brain tissue [1], as approximately 90\% of the brain non-heme iron is normally bound to ferritin. Other endogenous paramagnetic materials (e.g. copper and manganese) are considered to have insufficient concentrations in healthy individuals to affect the MRI signal. Therefore, MRI methods focus on the greatest localised source of non-heme iron: ferritin $[1,5]$.

The presence of iron atoms inside ferritin molecules leads to the formation of ferric oxyhydroxide particles, which has an influence on the final contrast of the $T 2$ weighted MR images; therefore, ferritin and hemosiderin are thought to be the most important sources of iron related to signal changes in cerebral MRI $[1,3]$. Such iron causes hypointensive artifacts, which have the potential to become a biomarker of neural iron-related disorders.

With the advent of high-field scanners and ironsensitive sequences such as $T 2 *$ weighted imaging $(T 2 * \mathrm{WI})$, susceptibility weighted imaging or fielddependent relaxivity increase, MRI has become an indispensable diagnostic and research tool in the study of disorders involving iron disregulation.

The aim of this study is to investigate whether it is possible with standard $T 2$ weighted MRI protocols to distinguish between healthy and neuropathological tissue. The findings of Khalil et al. reveal that iron accumulation in 
the basal ganglia is more pronounced in the early rather than later phases of disease, and occurs independently of other morphological brain changes [6]; this can therefore be used in early diagnostics of the initial phases of disorders associated with disrupted iron homeostasis.

\section{Cases}

Nineteen healthy volunteers (13 females, aged $42.92 \pm 10.81$ years; 6 males aged $54.33 \pm 17.60$ years) were included in the study, as well as 13 patients ( 7 females, aged $42.00 \pm 14.30$ years; 6 males aged $41.17 \pm 15.63$ years) with multiple sclerosis or the Parkinson disease.

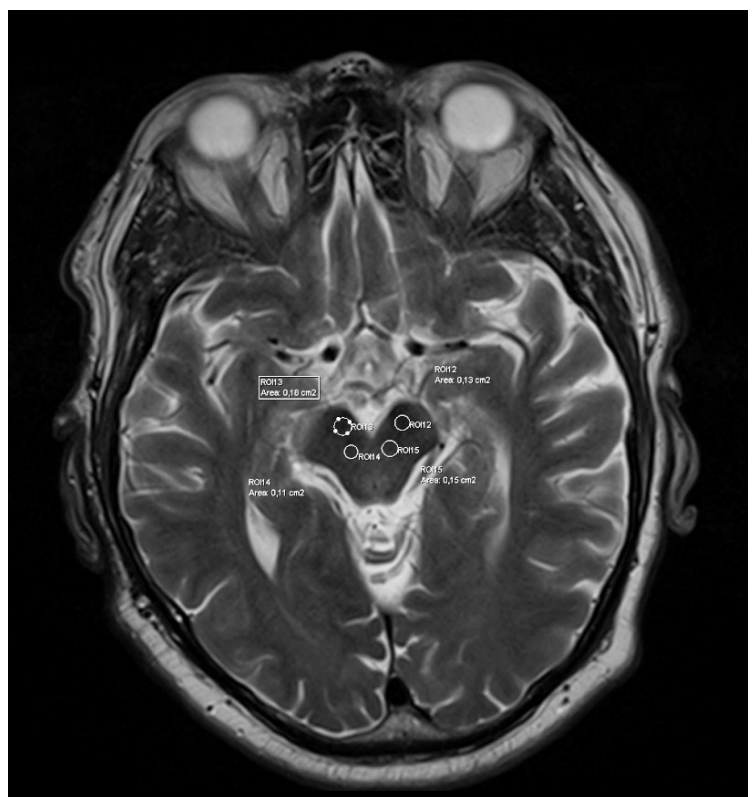

Fig. 1. T2 clinical MRI data. Regions of interest (4 ring area) in the basal ganglia marked by the radiologists.

Clinical MRI data were provided by Prof. Bořuta from the Slovak Medical University, Bratislava (Fig. 1). All subjects underwent MR examination in a Siemens Magnetom Verio 3T system (Siemens HealthCare, Erlangen, Germany) by standard T2 weighted gradient-echo sequence. The mean value of the absolute intensity of the investigated region of interest (ROI) in the basal ganglia or related structures was compared with the reference ROI in grey matter for each subject and each slice, and for both the patients and group of healthy volunteers (Fig. 2).

The final relative $T 2$ contrast for every person, every slice, and every ROI was calculated as follows:

$$
R C=\frac{\left(c-c_{0}\right)}{c_{0}},
$$

where $R C$ is the relative contrast, $c$ represents the mean contrast of each sample ROI in the MRI image slice, and $c_{0}$ is the grand mean [7] contrast of all reference ROIs in the same MRI image slice. T2 relative contrast control and pathological group is shown in Fig. 3.

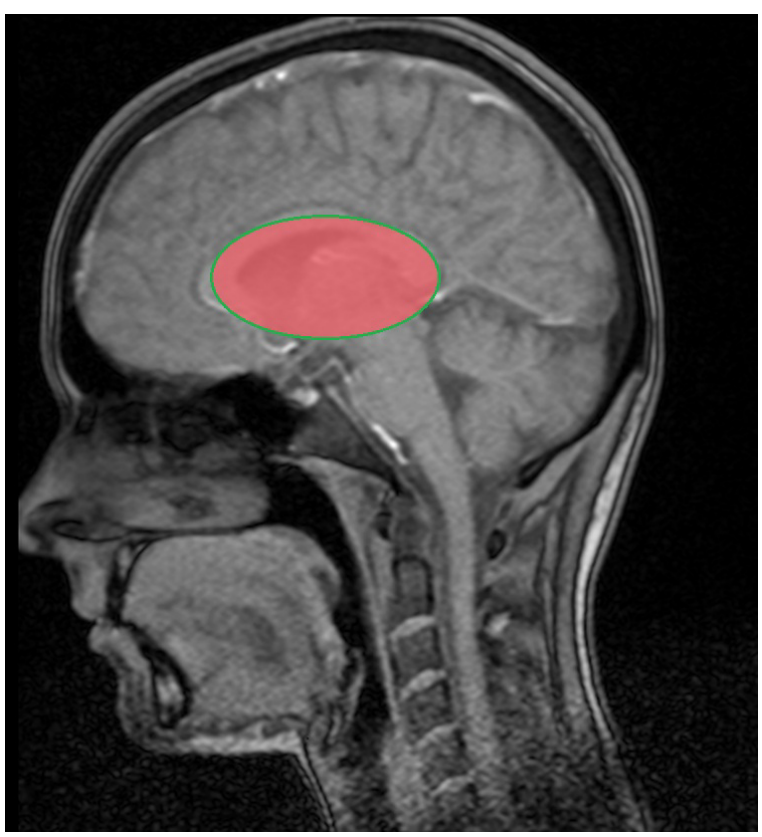

Fig. 2. MR image of human brain, with basal ganglia and related structures highlighted.
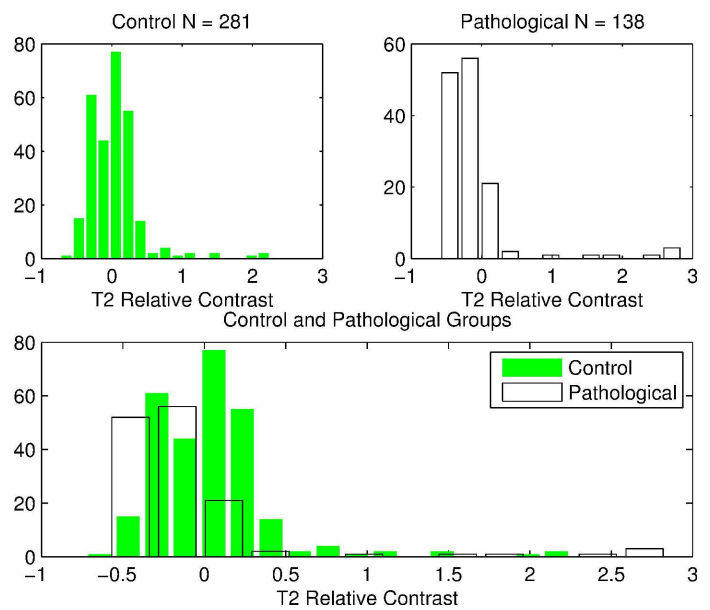

Fig. 3. T2 relative contrast control and pathological group.

Statistical tests were applied to determine the type of data distribution (the Shapiro-Wilk) and the signal intensity difference between the volunteers and patients (the Kruskal-Wallis). A $p$-value of less than 0.05 was considered statistically significant.

\section{Results and discussion}

Our results indicate that we were able to distinguish between the healthy and diseased groups in a statistically significantly manner. The Shapiro-Wilk test showed that neither group was distributed normally, while the subsequent non-parametric Kruskal-Wallis test confirmed 
that both investigated groups were significantly different $(p<0.001)$. Statistical evaluation of the standard $T 2$ weighted MR images of selected brain tissue objects revealed significant difference between the control (without neuropathology) and pathological group, using the non-parametric statistical method $\left(p=8.7746 \times 10^{-11}\right)$. These preliminary findings suggest that MRI is a feasible diagnostic tool for multiple sclerosis and the Parkinson disease. Moreover, these results might be further employed in the research of iron quantification and the non-invasive diagnostics of neuropathological diseases associated with the accumulation of iron in brain tissue.

\section{Conclusions}

Statistical evaluation of standard $T 2$ weighted MR images of selected brain tissue objects presented significant difference between the control (without neuropathology) and pathological group, using the non-parametric statistical method. Based on these limited data it seems feasible that a diagnostic tool for several iron-sensitive neurodegenerative conditions could be developed. The outcome of this study thus represents our starting point for further research in the field of iron quantification and non-invasive diagnostics of neuropathological diseases associated with the accumulation of iron in brain tissue.

\section{Acknowledgments}

This work was supported by the Slovak Research and Development Agency APVV-0431-12, APVV-150029 and by the Slovak Scientific Grant Agency VEGA $2 / 0013 / 14$.

\section{References}

[1] P. Dusek, M. Dezortova, J. Wuerfel, Int. Rev. Neurobiol. 110, 195 (2013).

[2] C.B. Sirlin, S.B. Reeder, Magn. Reson. Imaging Clin. N. Am. 18, 359 (2010).

[3] G. Bartzokis, D. Sultzer, J. Cummings, L.E. Holt, D.B. Hance, V.W. Henderson, J. Mintz, Arch. Gen. Psychiatry 57, 47 (2000).

[4] L.H. You, F. Li, L. Wang, S.E. Zhao, S.M. Wang, L.L. Zhang, L.H. Zhang, X.L. Duan, P. Yu, Y.Z. Chang, Neuroscience 284, 234 (2015).

[5] J. Galazka-Friedman, A. Friedman, E.R. Bauminger, Hyperfine Interact. 189, 31 (2009).

[6] M. Khalil, C. Langkammer, A. Pichler, D. Pinter, T. Gattringer, G. Bachmaier, S. Ropele, S. Fuchs, C. Enzinger, F. Fazekas, Neurology 84, 24 (2015).

[7] D.A. Burton, Composite Standard Deviations, (accessed: 30 May 2016). 